\title{
SPATIAL AND SPECTRAL STRUCTURE OF CHROMOSPHERIC LINES
}

\author{
JAY M. PASACHOFF and FREDERICK S. HARRIS \\ Williams College-Hopkins Observatory, Williamstown, Mass. 01267, U.S.A. \\ and \\ JACQUES M. BECKERS \\ Sacramento Peak Observatory, AFCRL, Sunspot, N.M. 88349, U.S.A.
}

\begin{abstract}
We are reducing a set of spectra covering the region from 3400 to $4330 \AA$ that show both spatial and spectral structure in chromospheric emission lines from many elements and ions. The spectra were taken with the vacuum tower telescope at the Sacramento Peak Observatory with the spectrograph slit tangent to and touching the limb. Thus some height resolution in the chromosphere is present. Many of the lines show doubly-reversed emission, often asymmetric either for the whole line or for fine structure. The quality of the data is such as to improve our understanding of line blends. The spectral structure can be compared with the well-known structure in the $\mathrm{H}$ and $\mathrm{K}$ lines of $\mathrm{Ca}$ II and in the resonance lines of $\mathrm{MgII}$ in order to deduce a model for the lower chromosphere.
\end{abstract}

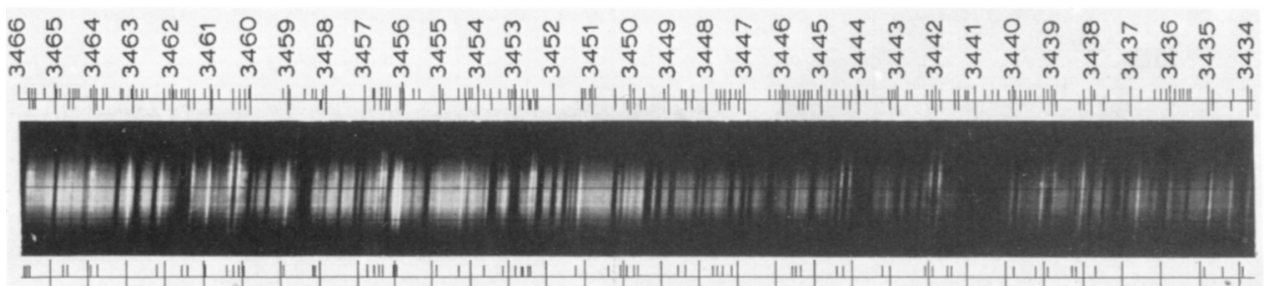

Fig. 1. Part of one of the spectra that are being analyzed for the new chromospheric atlas. Tick marks facing inwards towards the spectra on the top and bottom are lines identified on this print; tick marks facing outward represent lines reported in the atlas of Pierce. This sample covers the region from 3434 to 3465. As the center of the slit is slightly lower down than the edges, it shows photospheric continuum in addition to the emission lines.

Many rare-Earth lines are also visible in emission on the spectra and, contrary to the other lines, show no spatial or spectral structure even under this high resolution.

The wavelength region is covered with 13 separate spectra, each covering about $70 \AA$ at dispersions ranging from 9 to $13 \mathrm{~mm} \AA^{-1}$. About 200 emission lines are visible on the best of the plates.

An atlas is being compiled from these spectra that will comment on the spatial and spectral structure, for comparison with the atlas of Pierce. 


\section{DISCUSSION}

(i) On the Reality of the Central Reversal

Beckers questioned the origin of the self reversed profiles. In similar spectra taken at the solar limb the central reversal disappeared after the scattered light was subtracted. Are all central reversals due to scattered light (Zirin)? No, especially eclipse spectra show many self reversed lines which must be real (Jefferies, Pierce, Pasachoff and Rutten). Beckers persists in questioning the reality of many of the reversals in the spectra presented by Pasachoff. 